\title{
Periodic Variation of Solar Flare Index for the Last Solar Cycle (Cycle 24)
}

\author{
Atila Ozguc $\mathbb{D}^{1},{ }^{1}$ Ali Kilcik $\mathbb{D}^{2},{ }^{2}$ Volkan Sarp $\mathbb{D}^{\circ},{ }^{2}$ Hülya Yeşilyaprak, ${ }^{1}$ and Rıza Pektaş ${ }^{1}$ \\ ${ }^{1}$ Kandilli Observatory and Earthquake Research Institute, Bogazici University, Istanbul 34684, Turkey \\ ${ }^{2}$ Department of Space Science and Technologies, Akdeniz University, Faculty of Science, Antalya 07058, Turkey
}

Correspondence should be addressed to Volkan Sarp; volkansarp@akdeniz.edu.tr

Received 6 May 2021; Accepted 15 August 2021; Published 24 August 2021

Academic Editor: Fernando Aguado Agelet

Copyright (c) 2021 Atila Ozguc et al. This is an open access article distributed under the Creative Commons Attribution License, which permits unrestricted use, distribution, and reproduction in any medium, provided the original work is properly cited.

In this study, we used the flare index (FI) data taken from Kandilli Observatory for the period of 2009-2020. The data sets are analyzed in three categories as Northern Hemisphere, Southern Hemisphere, and total FI data sets. Total FI data set is obtained from the sum of Northern and Southern Hemispheric values. In this study, the periodic variations of abovementioned three categories FI data sets were investigated by using the MTM and Morlet wavelet analysis methods. The wavelet coherence (XWT) and cross wavelet (WTC) analysis methods were also performed between these data sets. As a result of our analysis, the following results were found: (1) long- and short-term periodicities (2048 \pm 512 day and periodicities smaller than 62 days) exist in all data sets without any exception at least with $95 \%$ confidence level; (2) all periodic variations were detected maximum during the solar cycle, while during the minima, no meaningful period is detected; (3) some periodicities have data preference that about 150 days Rieger period appears only in the whole data set and 682-, 204-, and 76.6-day periods appear only in the Northern Hemisphere data sets; (4) During the Solar Cycle 24, more flare activity is seen at the Southern Hemisphere, so the whole disk data periodicities are dominated by this hemisphere; (5) in general, there is a phase mixing between Northern and Southern Hemisphere FI data, except about 1024-day periodicity, and the best phase coherency is obtained between the Southern Hemisphere and total flare index data sets; (6) in case of the Northern and Southern Hemisphere FI data sets, there is no significant correlation between two continuous wavelet transforms, but the strongest correlation is obtained for the total FI and Southern Hemisphere data sets.

\section{Introduction}

Solar flares (SFs) are one of the most intense eruptions observed on the Sun. They can occur in the corona above active regions, sunspots, magnetic network boundaries, network, etc. [1-3]. Large flares mainly occur in the complex active regions that they have magnetically complex structure. The duration of SFs can vary from a few seconds to a few hours. During an SF, energy is released in all wavelengths of the electromagnetic spectrum from gamma to radio waves. The amount of released total energy during a large flare is about $10^{32} \mathrm{erg}$. During the flaring activity, solar energetic particles such as electrons, protons, and heavy nuclei are also released and accelerated $[4,5]$. Especially large flares may produce intense effects on
Earth and near space environment $[3,6,7]$. Therefore, the regular observations of solar flares have very big importance, especially from the point of space weather and Sun-Earth interactions.

Since the first SF observation made by Carrington and Hodgson in 1859 in the continuum of white light as a relatively short brightening increase [3], which lasts on the order of minutes, began to attract the attention of scientists working on the solar physics and space weather and even more, they were regularly monitored in the hydrogen alpha $(\mathrm{H} \alpha ; 6563 \AA)$ which is the chromospheric line and these observations are still ongoing. Since the satellite age began, in addition to the $\mathrm{H} \alpha$ observations, they started to observe from the space in X-rays via satellites. 
To measure the amount of total energy emitted by the solar flare in the $\mathrm{H} \alpha$ wavelength (6563 $\AA$ ), flare index (FI) which describes the short lived solar activity was introduced in [8];

$$
\mathrm{FI}=i t
$$

where $i$ represents the importance scale of a flare in $\mathrm{H} \alpha$ and it is based on the size and brightness of the flaring area and $t$ describes the duration (in minutes) of the flare (for detail see, [9]).

The temporal variation of FI follows the general solar activity variation. Therefore, they are strongly related to all other solar activity indicators such as sunspot number, $10.7 \mathrm{~cm}$ solar radio flux, and total solar irradiance. Similar to other solar activity indicators, FI also shows periodic behavior from days to tens of years and it is a suitable index to study short and long range periodic behavior of solar activity. The periodic variation of solar activity indices is investigated by using different indicators such as sunspot number and area, TSI, $10.7 \mathrm{~cm}$ solar radio flux, and flare index $[5,10-12]$. The well-known periodic variations are the 27-day synodic rotation and 11-year Schwabe Cycle due to solar internal dynamo $[9,13]$. Apart from these two, the 51-, 75-, 154-, and 323-day and 1.3-, 1.8-, and 3.5-year periodicities are also reported in the literature $[5,10,12,14-16]$.

It is known that active structures observed on the Sun are distributed unevenly between the Northern and the Southern Hemispheres of it and this phenomenon is called north-south asymmetry. This asymmetry is observed in data sets of sunspot number, sunspot area, sunspot group number, solar FI, and so on, and widely studied in the literature [12, 17-19]. Flare index was investigated temporarily and periodically before for the Cycles 20, 21, 22, and 23 [20-22]. Here, we investigated the periodic variation of solar FI data sets for the complete Solar Cycle 24 (from 2009 to 2020) by using the multitaper method (MTM) and Morlet wavelet analysis methods and we also obtained wavelet coherence (WTC) and cross wavelet (XWT) spectrums. We found most of the abovementioned periodicities exist in the FI data of Solar Cycle 24 and also we confirm the hemispheric asymmetry of the FI data that Northern and Southern Hemispheres data periodicities show some differences. The remainder of this paper is organized as follows: we describe data and methods in Section 2, our results are given in Section 3, and our discussion and conclusions are presented in Section 4.

\section{Data and Methods}

We investigated the periodic variation of the daily solar FI data within the period range between 25 and 2730 days by studying the complete Solar Cycle 24 (from 2009 to 2020). This time interval contains 4383 daily data points for each of the Northern Hemisphere, Southern Hemisphere, and whole disk which is produced by taking the sum of each hemispheric values. The data are taken from Boğaziçi University Kandilli Observatory (https://astronomi.boun.edu.tr/flare-index).

To investigate the periodic behavior of FI data, multitaper method (MTM) and Morlet wavelet analysis methods were used. The MTM provides useful tools for the spectral estimation $[23,24]$ and signal reconstruction [25] of a time series whose spectrum may contain both broadband and line components. It is a nonparametric method and it reduces the variance of spectral estimates by using a small set of tapers. It used to detect low-amplitude harmonic oscillations which have a high degree of statistical significance, especially in a given short and noisy as well as multivariate time series. To obtain an estimate of the power spectrum, the method uses tapers or orthogonal windows (for detail see, [26]). It has advantage compared to the other classical methods that it can reject larger amplitude harmonics if the F-test fails. It has been successfully used previously for the analysis of different data sets $[12,26-30]$. Here, we used three sinusoidal tapers, and the frequency range was chosen between 0.00037 and 0.04 (2730-25 days). The significance test was carried out assuming that the noise has a red spectrum. We considered that a signal was detected when the $95 \%$ confidence level was reached.

Morlet wavelet analysis is a valuable method to analyze localized variation of periodicities within a given time series $[31,32]$. In contrast to classical Fourier analysis, the wavelet analysis presents detected meaningful periods together with their possible temporal variations. It has been widely used in astronomical [12, 30, 33] and geophysical applications [32]. Here, to obtain the temporal variations of detected meaningful periods, MTM and the Morlet wavelet analysis method were used. In this study, daily FI data were analyzed using the "biwavelet package" written in $\mathrm{R}$ programming language [34]. "Morlet" mother function considering a "red noise" background with a nondimensional frequency was used [32]. The effect of edges is represented by the cone of influence (COI).

We also used cross wavelet transform (XWT) and wavelet coherence (WTC) analysis methods which are also given in the "biwavelet package." These two analysis methods present nonlinear relation and common periods between selected two data sets. The XWT spectrum shows the phase relation that is represented by arrows with the following manner: pointing right, in-phase; pointing left, antiphase; pointing straight up, the second series (Y) leads by $90^{\circ}$; pointing straight down, the first series $(X)$ leads by $90^{\circ}$. If the arrows are distributed randomly, it shows phase mixing between two time series (X and Y). The WTC spectrum indicates the amount of common power between the two time series as a function of time and frequency. It measures the cross correlation between two time series, even though the obtained common power is low [12, 35-37]. Here, we compared total FI data with the other solar activity indices given above.

\section{Results}

In Figure 1, we plotted the temporal variations of the used daily data sets. As shown in this figure, Northern and Southern Hemisphere FI data show some differences that the Northern Hemisphere is dominant during the first half of the cycle, while the Southern Hemisphere data become dominant during the second half of the cycle.

In Figures 2-4, we plotted the MTM and Morlet wavelet period analysis results of Northern Hemisphere, Southern Hemisphere, and whole disk FI data sets. In the MTM plots, 

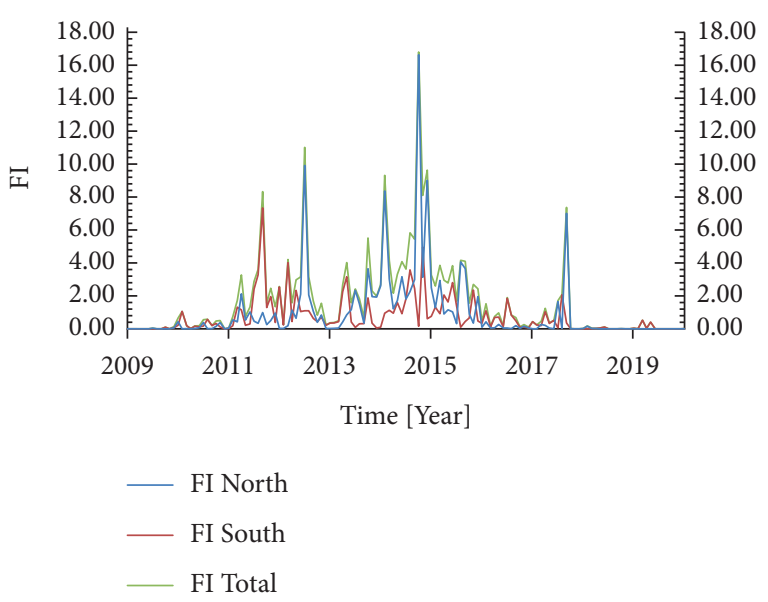

FIGURE 1: Temporal variation of the monthly FI data sets for the investigated time period. All data sets are smoothed by using the 12-step running average.
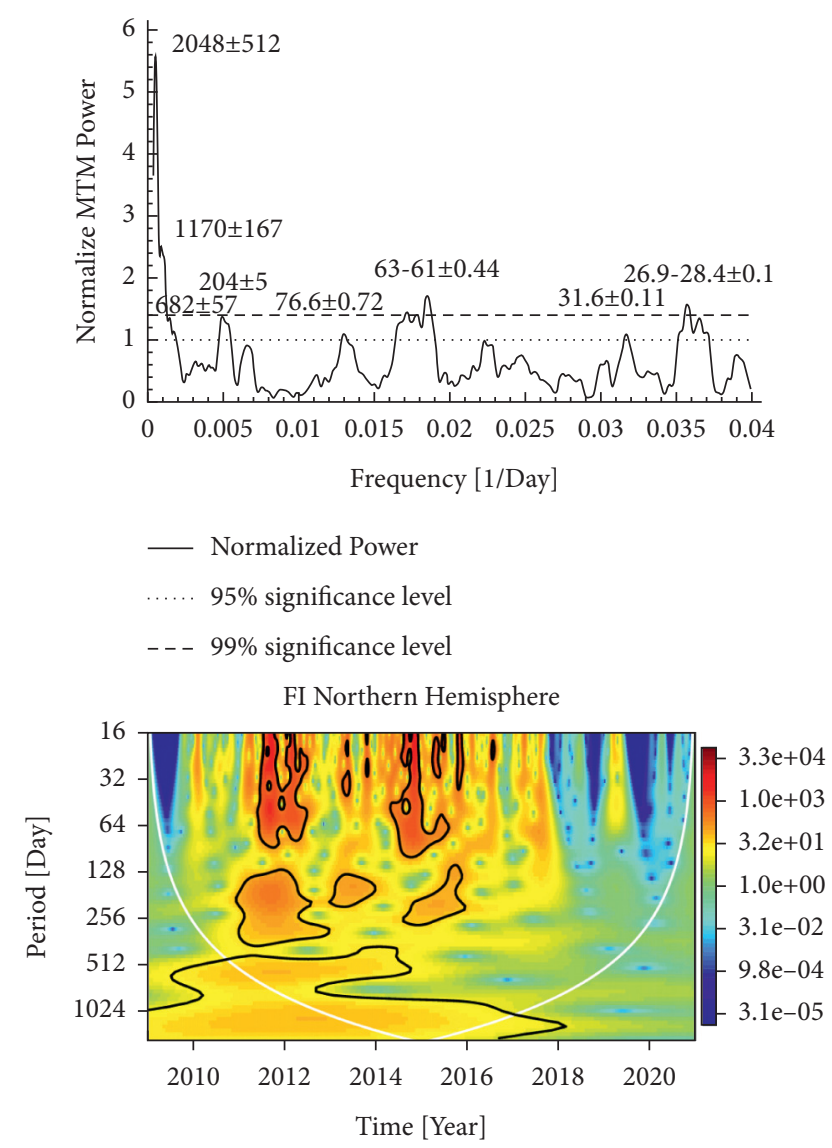

FIGURe 2: MTM (upper panel) and Morlet wavelet (lower panel) analysis results for Northern Hemisphere FI data for the investigated time interval. White solid line in the wavelet scalogram shows the cone of influence (COI); black solid lines show the significant periodicities.

horizontal dashed and long dashed lines represent 95\% and $98 \%$ confidence levels. All peaks exceeding the dashed line show the meaningful periods with at least $95 \%$ confidence
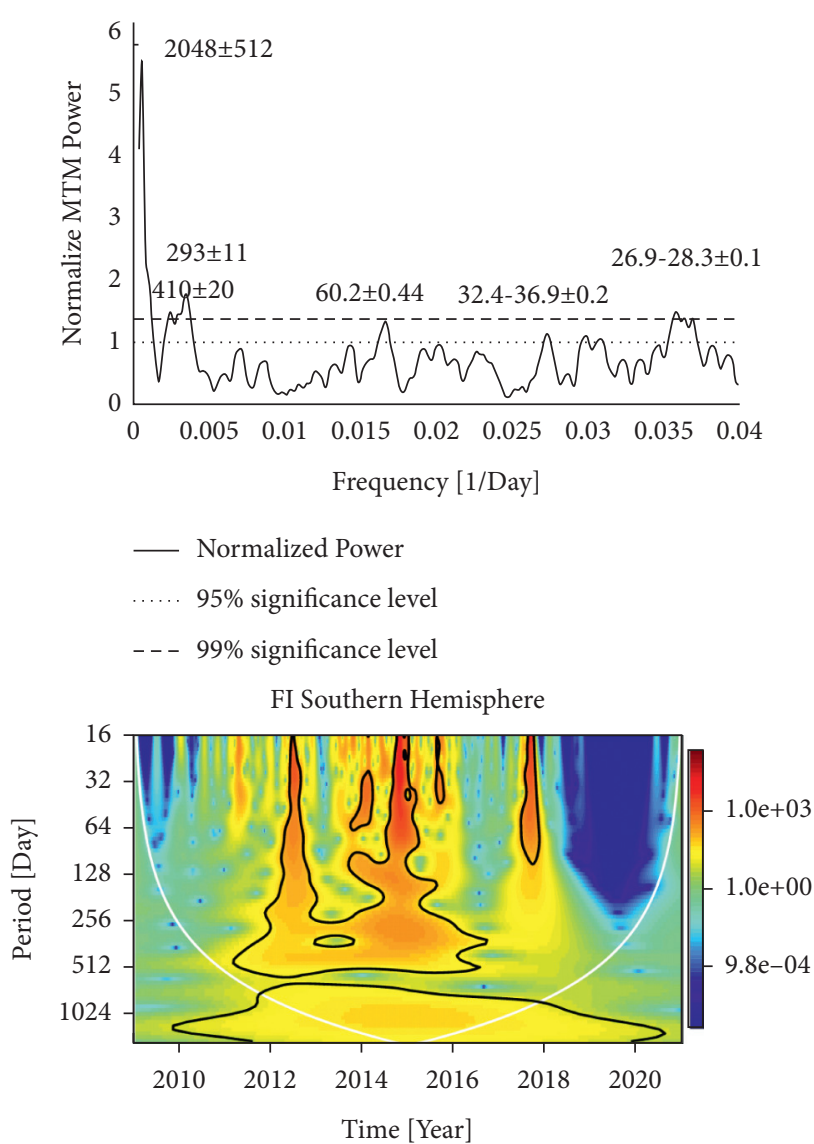

Figure 3: MTM (upper panel) and Morlet wavelet (lower panel) analysis results for Southern Hemisphere FI data for the investigated time interval. White solid line in the wavelet scalogram shows the cone of influence (COI); black solid lines show the significant periodicities.

level. In the Morlet wavelet scalogram, the significance of possible oscillation increased from blue to red color. The white curve called cone of influence (COI) that the periods located in the outside of this curve is not meaningful with 95\% confidence and black contours located inside the COI show the meaningful periods. In Table 1, we listed obtained meaningful periods, their existence, and significance levels. We present the XWT and WCT plots in Figures 5-7.

From these figures (Figures 2-4) and from Table 1, we obtained the following results: (i) $2048 \pm 512$ days (5.6 years) and the periodicities smaller than 62 days exist in all data sets without any exception at least with 95\% confidence level; (ii) as shown in Table 1, all periods appear during the solar cycle maximum; (iii) about 150 days Rieger period appears only in the whole data set, while 682-, 204-, and 76.6-day periods appear only in the Northern Hemisphere data sets; (iv) in general periodicities obtained from the Southern Hemisphere FI data are all appear in the whole disk data, therefore we may argue that the Southern Hemisphere flare activity was dominant during the Solar Cycle 24.

In the XWT plots, the black solid lines show the meaningful coherence between selected time series and arrows show their phase relations. As shown in these plots, 

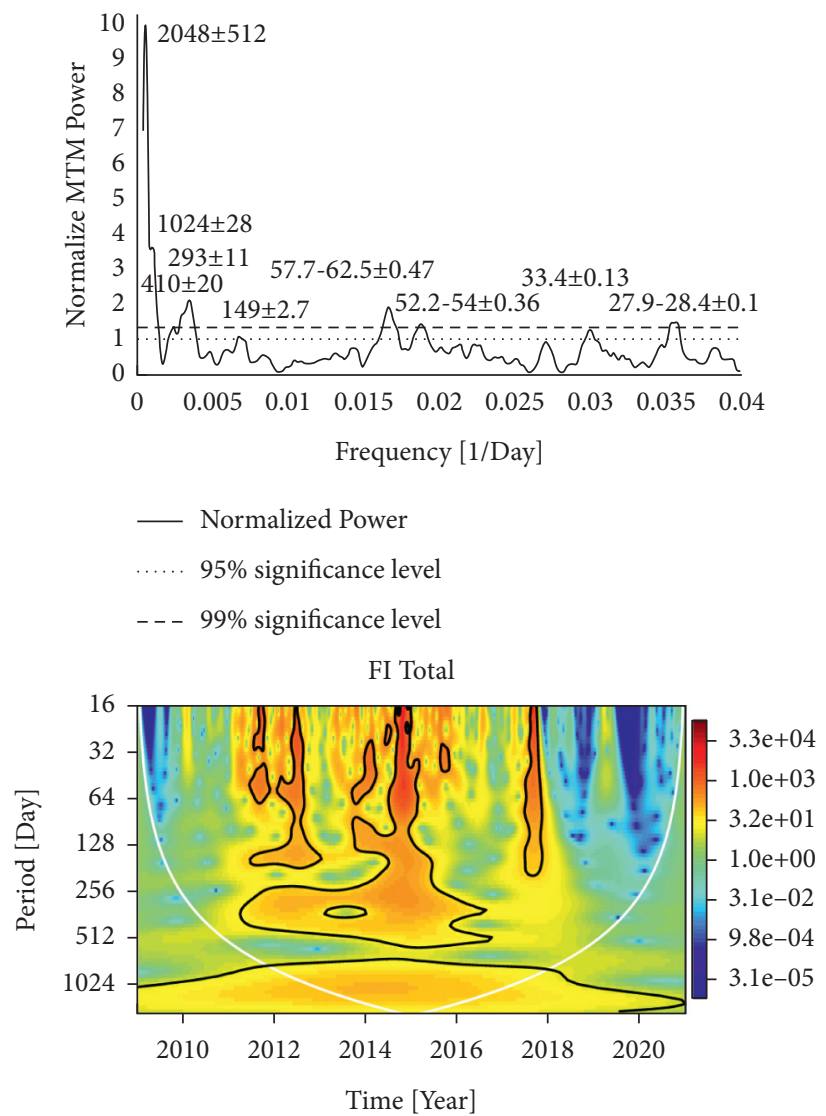

Figure 4: MTM (upper panel) and Morlet wavelet (lower panel) analysis results for whole disk FI data for the investigated time interval. White solid line in the wavelet scalogram shows the cone of influence (COI); black solid lines show the significant periodicities.

TABLE 1: Detected periods, their existence, and significance level in each data set used in this study.

\begin{tabular}{lcccc}
\hline Period (day) & Northern Hemisphere & Southern Hemisphere (\%) & Whole disk (\%) & Phase of the cycle \\
\hline 2048 & plusmn; 512 & $+>99$ & $+>99$ & $+>99 \%$ Max \\
1024 & plusmn; 128 & $+>99$ & - & $->99 \%$ Max \\
682 & plusmn; 57 & $+>95$ & $+>99$ & - first half \\
410 & plusmn; 20 & - & $+>99$ & $+>95 \%$ Max \\
293 & plusmn; 11 & - & - & $+>99 \%$ Max \\
204 & plusmn; 5 & $+>95$ & - & - Max \\
149 & plusmn; 2.7 & - & $+>99$ & $+>95 \%$ Max and descending \\
76.6 & plusmn; 0.72 & $+>95$ & $+>95$ & + Max \\
$52.2-62.5$ & plusmn; 0.44 & $+>99$ & $+>99 \%$ Max and descending \\
$26.9-36.9$ & plusmn; 0.2 & $+>95$ & Max and descending \\
\hline
\end{tabular}

in general there is a phase mixing between Northern and Southern Hemispheres FI data, except about 1024-day periodicity. When we look at the cross wavelet plot between whole disk and Northern Hemisphere FI (total FI and Northern Hemisphere FI) 16- and 76-day periodicities are in phase up to 2014, then the phase is mixed. The situation is completely different for the case of total FI and Southern Hemisphere. In this case, all periodic variations are in phase during the whole cycle. Thus, we may conclude that Southern Hemisphere FI is dominant compared to the Northern Hemisphere FI during the investigated cycle. From the WTC plots, we obtained the following results: there is no strong correlation between two continuous wavelet transforms in case of the Northern and Southern Hemispheres. The correlation gets much stronger for total FI and Northern Hemisphere FI cases, especially for the short and long periods. All periods are strongly correlated in case of the total FI and Southern Hemisphere FI for the investigated time interval.

\section{Discussion and Conclusion}

In this study, we analyzed the Northern and Southern Hemispheres and also total FI data periodic variations by using the MTM and Morlet wavelet analysis methods. We found the following results: 

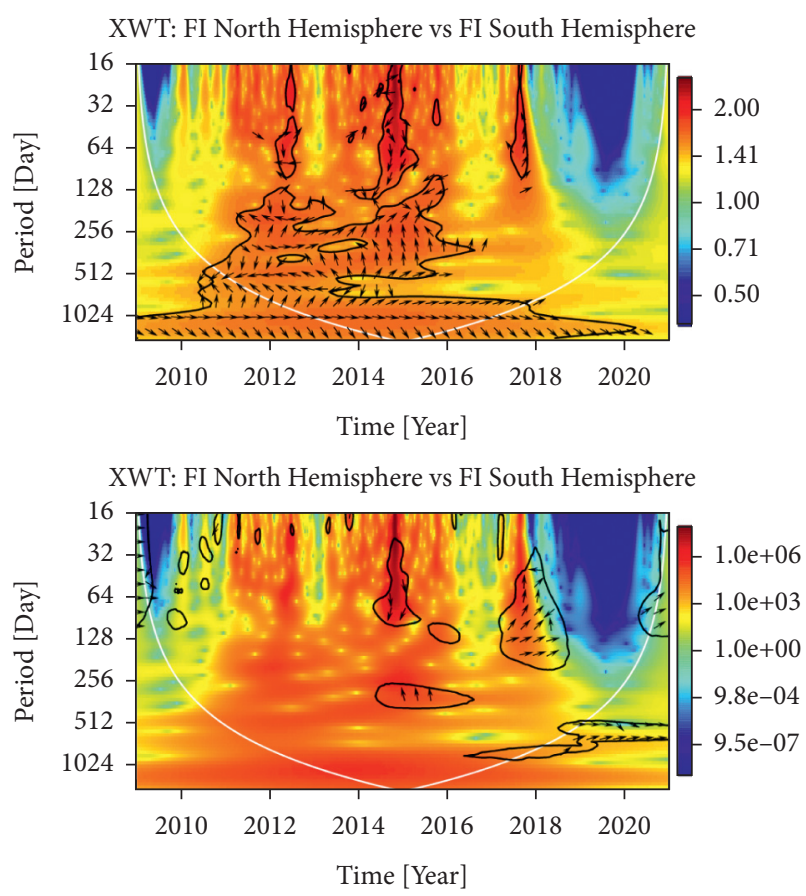

FIGURE 5: XWT (upper panel) and WTC (lower panel) spectrums between Northern and Southern Hemisphere FI data sets. The red color represents regions with the most significant interrelation, while blue colors show the lowest dependence between the selected time series. Periodicities obtained outside of the COI for all wavelet plots are usually not reliable. Arrows in the wavelet coherence plots represent the phase relations between the time series. A zero phase difference means that the two time series move together on a particular scale. Arrows point to the right/left when the time series are in phase (antiphase). Arrows pointing to the right-down or leftup indicate that the first variable is leading, while arrows pointing to the right-up or left-down show that the second variable is leading.

(i) Long- and short-term periodicities (2048 \pm 512 days and periodicities smaller than 62 days) exist in all data sets without any exception at least with $95 \%$ confidence level.

(ii) All periodic variations are detected during the solar cycle maximum, while during the minimum, no meaningful period is detected.

(iii) Some periodicities have data preference that about 150 days Rieger period, which appears only in the whole data set, 682-, 204-, and 76.6-day periods appear only in the Northern Hemisphere data sets.

(iv) Southern Hemisphere flare activity is dominant during the Solar Cycle 24 that, in general, periodicities obtained from the Southern Hemisphere FI data all appear in the whole disk data.

(v) In general, there is a phase mixing between Northern and Southern Hemisphere FI data, except about 1024-day periodicity, the best phase coherency is obtained between the Southern Hemisphere and total flare index data sets.

(vi) There is no strong correlation between two continuous wavelet transforms in case of the Northern
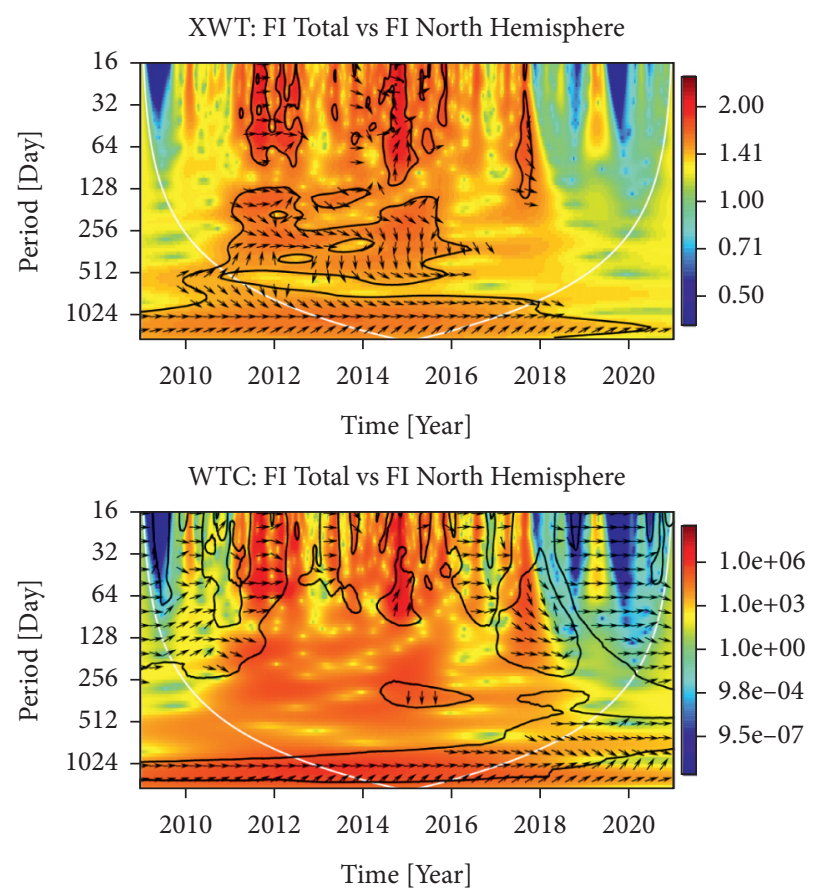

FIGURE 6: XWT (upper panel) and WTC (lower panel) spectrums between whole disk and Northern Hemisphere FI data. The red color represents regions with the most significant interrelation, while blue colors show the lowest dependence between the selected time series. Periodicities obtained outside of the COI for all wavelet plots are usually not reliable. Arrows in the wavelet coherence plots represent the phase relations between the time series. A zero phase difference means that the two time series move together on a particular scale. Arrows point to the right/left when the time series are in phase (antiphase). Arrows pointing to the right-down or leftup indicate that the first variable is leading, while arrows pointing to the right-up or left-down show that the second variable is leading.

and Southern Hemisphere FI data sets. The best correlation is obtained for the total FI and Southern Hemisphere.

We found $2048 \pm 512,1024 \pm 128,682 \pm 57,410 \pm 20$, $293 \pm 11,204 \pm 5,149 \pm 2.7,76.6 \pm 0.72,52.2-62.5 \pm 0.44$, and 26.9-36.9 \pm 0.2 -day periodicities from our MTM and Morlet wavelet analysis results with at least $95 \%$ confidence level. Almost all of these periodicities $(1024,682,410,293$, 204,149 , and 76.6 days) have hemispheric preference; the others $(2048,52.2-62.5$, and 26.9-36.9) do not have and they exist in all data sets used in this study. Kilcik et al. [10] investigated the periodicity of the daily whole disk FI data from Cycle 21 to 23. They used MTM and Morlet wavelet analysis methods and found that 25-37 days solar rotation periodicities exist in all cycle data without any exception. These periods describe the solar rotation and include all effects such as heliographic latitude, proper motion, and flaring effect acting on sunspot groups. 410-day periodicity is detected only in Solar Cycle 23, 682-day periodicity only in whole data, 149-day in whole data and in Cycle 21, and 76day in whole data, in Cycle 21, and in Cycle 22 and 52- and 62 -day periodicities in whole data and in Cycle 22 data sets. 

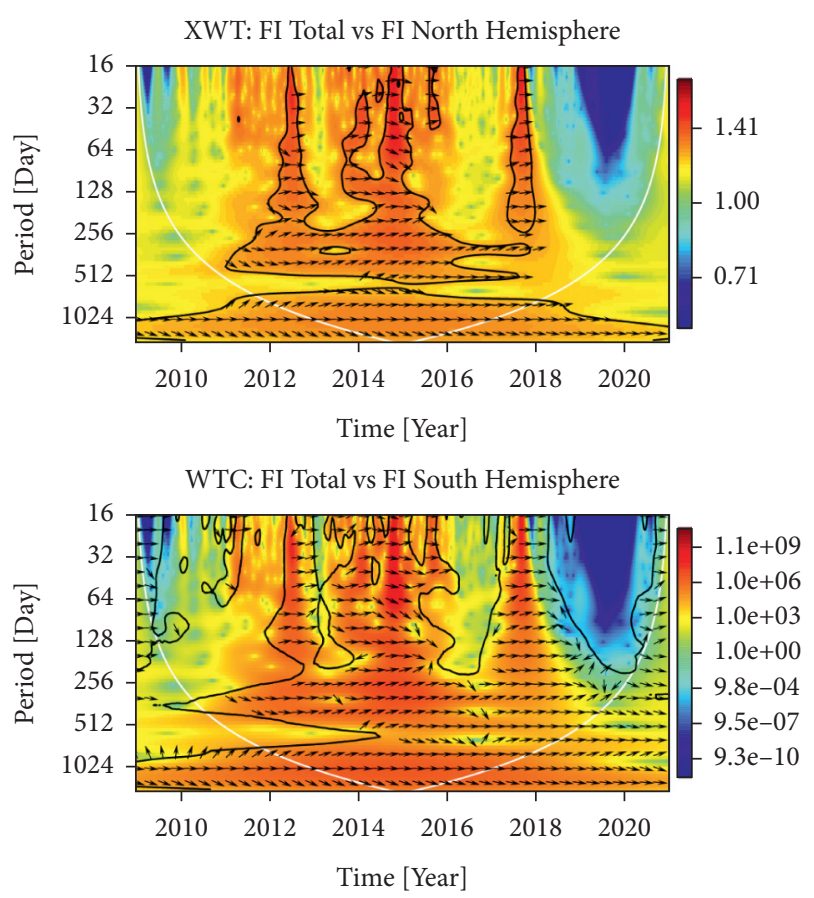

FIgURE 7: XWT (upper panel) and WTC (lower panel) spectrums between whole disk and Southern Hemisphere FI data. The red color represents regions with the most significant interrelation, while blue colors show the lowest dependence between the selected time series. Periodicities obtained outside of the COI for all wavelet plots are usually not reliable. Arrows in the wavelet coherence plots represent the phase relations between the time series. A zero phase difference means that the two time series move together on a particular scale. Arrows point to the right/left when the time series are in phase (antiphase). Arrows pointing to the right-down or leftup indicate that the first variable is leading, while arrows pointing to the right-up or left-down show that the second variable is leading.

Later, Tsichla et al. [38] investigated the periodicity in some solar and geomagnetic activity indices by using the Lomb-Scargle algorithm and wavelet analysis methods for the time interval of 1965-2018. They detected 26.7 and 150 days and 1.1, 1.9, and 5.6 years periodicities from monthly data sets of SSN, interplanetary magnetic field, and geomagnetic Ap index. Recently, Roy et al. [5] analyzed the periodicities of monthly FI data taken from Kandilli Observatory for the time interval of 1976-2014. They used Rayleigh power spectrum analysis method and found 5.6, 1.9 , and 1.1 years and $28,152,260$, and 188 days periodicities. They concluded that the periods of about 85 and 311 days are integral multiplies of 28 days solar rotation period and about 653 days and 1077 days periods are subharmonic of 11-year Schwabe Cycle. They also conclude that the 5.6 years periodicity was caused by the enhanced power of second harmonic of 11-year solar activity cycle due to its asymmetric nature. Here, we found the similar periods from FI data sets used in this study with $95 \%$ significance level. It is clear seen from Figure 1 that the Northern Hemisphere is dominant during the first half of the Cycle 24 and then the Southern Hemisphere became dominant after 2014. Thus, we confirm the above findings and further suggest that the 5.6 years (2048 days) periodicity possibly come from the double peak structure of the solar cycle and it may be related to solar internal dynamo. Solar indices have a major 11-year cycle. However, some of the maxima of these indices were not smooth but structured. Two or more peaks can be identified during the solar maximum years. This splitting of activity was firstly identified in the green corona line intensity data by $[39,40]$ and named Gnevyshev gap. The double peaks are reported to be detected in all solar atmospheric layers (photosphere, chromosphere, and corona) up to interplanetary space and are linked with the heliomagnetic cycle. Feminella and Storini [41] reported that the long-term behavior of several solar activity parameters such as spots, flares, radio, and X-ray fluxes confirmed the double peaks and found that the peaks are more distinct with a clear gap in between when intense and/or long-lasting events are considered. Ahluwalia [42] found that solar polar field reversals may be responsible for the existence of the Gnevyshev gap in Ap data. Later on, it was reported for the most of the solar and interplanetary indices [43]. Kane [44] has reported this event by using sunspot numbers and solar electromagnetic radiations, namely, Lyman- $\alpha$, the $2800 \mathrm{MHz}$ solar radio emission, the coronal index, and mean magnetic field data as well as the geomagnetic indices Dst, $\mathrm{Ap}, \mathrm{AU}, \mathrm{AL}$, and AE during the rising phase of Solar Cycle 23. It is also observed that [45] the Cycle 24 is also a doublepeaked cycle, like the Cycle 23.

About 150 days periodicity detected in our study from the whole disc FI data is the Rieger periodicity which was first detected by [14] from the X-ray flare data. It was also detected by [5] as 152 days from the FI data and they argue that this periodicity appears due to the magnetic Rossby waves. Recently, Kilcik et al. [30] found the same periodicity from the MCMESI and different class X-ray solar flare data. The source of this periodicity is still unclear and many attempts were made in the literature. It may be related with magnetic flux emergence from deep solar interior [46] or it related with magnetic Rossby waves [5, 47-49].

Recently, Roy et al. [5] studied the north-south asymmetry of the monthly solar flare index during Solar Cycles 21-24 and found that the Northern Hemisphere is dominant during the first half of Cycle 24 and the Southern Hemisphere is dominant during the second half of the Cycle 24 . Our XWT and WTC plots exhibit the less small periods and the low correlation in time frequency space between the Northern and Southern Hemispheres during the Cycle 24. Also, the phase mixing is existing between these two data sets, except 1024-day periodicity. This result may indicate the hemispheric asymmetry. In case of the Northern Hemisphere and whole disc FI data, the high correlation exists in general and the phase mixing generally appears in the second half. For the Southern Hemisphere, generally high correlation exists and these two data sets are in phase during the Cycle 24. This result shows that the Southern Hemisphere is dominant during the investigated cycle. Thus, we confirm the above results and further obtained the asymmetric behavior from the XWT and WTC analysis. 


\section{Data Availability}

The FI data used in this study were taken from the Kandilli Observatory. The biwavelet package was provided by Tarik C. Gouhier and is available from https://github.com/ tgouhier/biwavelet.

\section{Conflicts of Interest}

The authors declare that they have no conflicts of interest.

\section{References}

[1] S. Krucker, A. O. Benz, T. S. Bastian, and L. W. Acton, "X-Ray network flares of the quiet sun," The Astrophysical Journal, vol. 488, no. 1, pp. 499-505, 1997.

[2] H. Li, Y. Liu, J. Liu, A. Elmhamdi, and A.-S. Kordi, "Two energy-release processes observed in the eruption of a confined filament system," Publications of the Astronomical Society of the Pacific, vol. 130, no. 994, Article ID 124401, 2018.

[3] A. O. Benz, "Flare observations," Living Reviews in Solar Physics, vol. 5, no. 1, 2008.

[4] I. A. Daglis, D. Delcourt, and F.-A. Kamide, "Particle acceleration in the frame of the storm-substorm relation," IEEE Transactions on Plasma Science, vol. 32, no. 4, pp. 1449-1454, 2004.

[5] S. Roy, A. Prasad, K. Ghosh, S. C. Panja, and S. N. Patra, "Chaos and periodicities in solar flare index from Kandilli observatory during 1976-2014," Research in Astronomy and Astrophysics, vol. 20, no. 7, p. 110, 2020.

[6] F. Shen, X. S. Feng, Y. Wang et al., "Three-dimensional MHD simulation of two coronal mass ejections' propagation and interaction using a successive magnetized plasma blobs model," Journal of Geophysical Research: Space Physics, vol. 116, no. A9, pp. a-n, 2011.

[7] L. Deng, X. Zhang, J. An, and Y. Cai, "Statistical properties of solar $\mathrm{H} \alpha$ flare activity," Journal of Space Weather and Space Climate, vol. 7, no. A34, 2017.

[8] J. Kleczek, "Ionospheric disturbances and flares in the 11years cycle," Bulletin of the Astronomical Institutes of Czechoslovakia, vol. 3, no. 52, 1952.

[9] A. Özgüç, T. Ataç, and J. Rybák, “'“Short-term periodicities in the flare index between the years 1966-2001" Solar variability as an input to the Earth's environment," in Proceedings of International Solar Cycle Studies (ISCS) Symposium, Tatranská Lomnica, Slovak Republic, June 2003.

[10] A. Kilcik, A. Özgüç, J. P. Rozelot, and T. Ataç, "Periodicities in solar flare index for cycles 21-23 revisited," Solar Physics, vol. 264, no. 1, pp. 255-268, 2010.

[11] D. H. Hathaway, "The solar cycle," Living Reviews in Solar Physics, vol. 12, no. 1, 2015.

[12] P. Chowdhury, A. Kilcik, V. Yurchyshyn, V. N. Obridko, and J. P. Rozelot, "Analysis of the hemispheric sunspot number time series for the solar cycles 18 to 24," Solar Physics, vol. 294, no. 10, 2019.

[13] L. H. Deng, B. Li, Y. Y. Xiang, and G. T. Dun, "Multi-scale analysis of coronal Fe xiv emission: the role of mid-range periodicities in the Sun-heliosphere connection," Journal of Atmospheric and Solar-Terrestrial Physics, vol. 122, pp. 18-25, 2015.

[14] E. Rieger, G. H. Share, D. J. Forrest, G. Kanbach, C. Reppin, and E. L. Chupp, "A 154-day periodicity in the occurrence of hard solar flares?” Nature, vol. 312, no. 5995, pp. 623-625, 1984.
[15] R. Oliver, M. Carbonell, and J. L. Ballester, "Intermediateterm periodicities in solar activity," Solar Physics, vol. 137, no. 1, pp. 141-153, 1992.

[16] T. Bai, "Periodicities in solar flare occurrence: analysis of cycles 19-23," The Astrophysical Journal, vol. 591, no. 1, pp. 406-415, 2003.

[17] T. Ataç and A. Özgüç, "North-South asymmetry in the solar flare index," Solar Physics, vol. 166, no. 1, p. 201, 1996.

[18] Y. Li, J. Jin, and H. Wang, "The change of magnetic inclination angles associated with flares," American Astronomical Society, vol. 41 , no. $850,2009$.

[19] J. Javaraiah, "North-south asymmetry in small and large sunspot group activity and violation of even-odd solar cycle rule," Astrophysics and Space Science, vol. 361, no. 7, p. 208, 2016.

[20] A. Özgüç and T. Ataç, "Periodic behavior of solar flare index during solar cycles 20 and 21," Solar Physics, vol. 123, no. 2, pp. 357-365, 1989.

[21] T. Ataç and A. Özgüç, "Flare index of solar cycle 22," Solar Physics, vol. 180, no. 1/2, pp. 397-407, 1998.

[22] T. Ataç and A. Özgüç, "Overview of the solar activity during solar cycle 23," Solar Physics, vol. 233, no. 1, pp. 139-153, 2006.

[23] D. J. Thomson, "Spectrum estimation and harmonic analysis," in Proceedings of the IEEE, vol. 70, no. 9, pp. 1055-1096, 1982.

[24] D. B. Percival and A. T. Walden, Spectral Analysis for Physical Applications, Cambridge University Press, Cambridge, UK, 1993.

[25] J. Park, "Envelope estimation for quasi-periodic geophysical signals in noise: a multitaper approach," in Statistics in the Environmental and Earth Sciences, A. T. Waldenand and P. Guttorp, Eds., pp. 189-219, Edward Arnold, London, UK, 1992.

[26] M. Ghil, M. R. Allen, M. D. Dettinger et al., "Advanced spectral methods for climatic time series," Reviews of Geophysics, vol. 40, no. 1, p. 1003, 2002.

[27] K. Fang, X. Gou, F. Chen et al., "Tree-ring based reconstruction of drought variability (1615-2009) in the Kongtong Mountain area, northern China," Global and Planetary Change, vol. 80-81, pp. 190-197, 2012.

[28] R. Escudier, J. Mignot, and D. Swingedouw, "A 20-year coupled ocean-sea ice-atmosphere variability mode in the North Atlantic in an AOGCM," Climate Dynamics, vol. 40, no. 3-4, pp. 619-636, 2012.

[29] A. Kilcik, V. Yurchyshyn, B. Donmez, V. N. Obridko, A. Özgüç, and J. P. Rozelot, "Temporal and periodic variations of sunspot counts in flaring and non-flaring active regions," Solar Physics, vol. 293, no. 4, p. 63, 2018.

[30] A. Kilcik, P. Chowdhury, V. Sarp et al., "Temporal and periodic variation of the MCMESI for the last two solar cycles; Comparison with the number of different class X-ray solar flares," Solar Physics, vol. 295, no. 11, p. 159, 2020.

[31] K.-M. Lau and H. Weng, "Climate signal detection using wavelet transform: how to make a time series sing," Bulletin of the American Meteorological Society, vol. 76, no. 12, pp. 2391-2402, 1995.

[32] C. Torrence and G. P. Compo, "A practical guide to wavelet analysis," Bulletin of the American Meteorological Society, vol. 79, no. 1, pp. 61-78, 1998.

[33] J. Oloketuyi, Y. Liu, and M. Zhao, "The periodic and temporal behaviors of solar X-ray flares in Solar cycles 23 and 24," The Astrophysical Journal, vol. 874, no. 1, p. 20, 2019.

[34] T. C. Gouhier, A. Grinsted, and V. Simko, R Package Biwavelet: Conduct Univariate and Bivariate Wavelet Analyses, https://github.com/tgouhier/biwavelet, 2018. 
[35] D. Maraun and J. Kurths, "Cross wavelet analysis: significance testing and pitfalls," Nonlinear Processes in Geophysics, vol. 11, no. 4, pp. 505-514, 2004.

[36] A. Grinsted, J. C. Moore, and S. Jevrejeva, “Application of the cross wavelet transform and wavelet coherence to geophysical time series," Nonlinear Processes in Geophysics, vol. 11, no. 5/6, pp. 561-566, 2004.

[37] C. Chang and G. H. Glover, "Time-frequency dynamics of resting-state brain connectivity measured with fMRI," $\mathrm{Neu}$ roImage, vol. 50, no. 1, pp. 81-98, 2010.

[38] M. Tsichla, M. Gerontidou, and H. Mavromichalaki, "Spectral analysis of solar and geomagnetic parameters in relation to cosmic-ray intensity for the time period 1965-2018," Solar Physics, vol. 294, no. 1, p. 15, 2019.

[39] M. N. Gnevyshev, "On the11-years cycle of solar activity," Solar Physics, vol. 1, pp. 107-120, 1967.

[40] M. N. Gnevyshev, "Essential features of the 11-year solar cycle," Solar Physics, vol. 51, pp. 175-183, 1977.

[41] F. Feminella and M. Storini, "Large-scale dynamical phenomena during solar activity cycles," Astronomy and Astrophysics, vol. 322, p. 311, 1997.

[42] H. S. Ahluwalia, "Aptime variations and interplanetary magnetic field intensity," Journal of Geophysical Research: Space Physics, vol. 105, no. A12, pp. 27481-27487, 2000.

[43] M. Storini, G. A. Bazilevskaya, E. O. Fluckiger, M. B. Krainev, V. S. Makhmutov, and A. I. Sladkova, "The GNEVYSHEV gap: a review for space weather," Advances in Space Research, vol. 31, no. 4, pp. 895-900, 2003.

[44] R. P. Kane, "Gnevyshev peaks in geomagnetic indices 2010," Planetary and Space Science, vol. 58, no. 5, 2010.

[45] V. K. Mishra and A. P. Mishra, "Long-term modulation of cosmic-ray intensity and correlation analysis using solar and heliospheric parameters," Solar Physics (2018), vol. 293, no. 10, p. 141, 2018.

[46] J. L. Ballester, R. Oliver, and M. Carbonell, "The near 160 day periodicity in the photospheric magnetic flux," The Astrophysical Journal, vol. 566, no. 1, pp. 505-511, 2002.

[47] M. Dimitropoulou, X. Moussas, and D. Strintzi, "Enhanced Rieger-type periodicities' detection in X-ray solar flares and statistical validation of Rossby waves' existence," Monthly Notices of the Royal Astronomical Society, vol. 386, no. 4, pp. 2278-2284, 2008.

[48] T. V. Zaqarashvili, M. Carbonell, R. Oliver, and J. L. Ballester, "Quasi-biennial oscillations in the solar tachocline caused by magnetic Rossby wave instabilities," The Astrophysical Journal, vol. 724, no. 1, pp. L95-L98, 2010.

[49] X. Feng, C. Li, C. Xiang, M. Zhang, H. Li, and F. Wei, "Datadriven modeling of the solar corona by a new three-dimensional path-conservative Osher-Solomon MHD model," The Astrophysical Journal-Supplement Series, vol. 233, no. 1, p. 10, 2017. 